\title{
Paternal germ line aging: DNA methylation age prediction from human sperm
}

Timothy G. Jenkins ${ }^{1 *}$, Kenneth I. Aston ${ }^{1}$, Bradley Cairns ${ }^{2}$, Andrew Smith ${ }^{3}$ and Douglas T. Carrell ${ }^{1,4}$

\begin{abstract}
Background: The relationship between aging and epigenetic profiles has been highlighted in many recent studies. Models using somatic cell methylomes to predict age have been successfully constructed. However, gamete aging is quite distinct and as such age prediction using sperm methylomes is ineffective with current techniques.

Results: We have produced a model that utilizes human sperm DNA methylation signatures to predict chronological age by utilizing methylation array data from a total of 329 samples. The dataset used for model construction includes infertile patients, sperm donors, and individuals from the general population. Our model is capable predicting age with an R2 of 0.89, a mean absolute error (MAE) of 2.04 years, and a mean absolute percent error (MAPE) of $6.28 \%$ in our data set. We additionally investigated the reproducibility of prediction with our model in an independent cohort where 6 technical replicates of 10 individual samples were tested on different arrays. We found very similar age prediction accuracy (MAE $=2.37$ years; $\mathrm{MAPE}=7.05 \%$ ) with a high degree of precision between replicates (standard deviation of only 0.877 years). Additionally, we found that smokers trended toward increased age profiles when compared to 'never smokers' though this pattern was only striking in a portion of the samples screened.

Conclusions: The predictive model described herein was built to offer researchers the ability to assess "germ line age" by accessing sperm DNA methylation signatures at genomic regions affected by age. Our data suggest that this model can predict an individual's chronological age with a high degree of accuracy regardless of fertility status and with a high degree of repeatability. Additionally, our data suggest that the aging process in sperm may be impacted by environmental factors, though this effect appears to be quite subtle and future work is needed to establish this relationship.
\end{abstract}

Keywords: Sperm epigenetics, Aging, DNA methylation, Aging calculator

\section{Background}

Recently, a great deal of work has been performed in an effort to understand the nature of aging, the mechanisms that drive the process, and the biomarkers that may be predictive of, or affected by, age. In this effort, a seminal manuscript was published in 2013 which described the ability to use DNA methylation signatures in somatic tissues to predict an individual's chronological age [1]. In this work, Dr. Horvath demonstrated that the epigenetic mechanisms that reflect the aging process are tightly conserved between individual tissues and across multiple species. Remarkably, these patterns are sufficiently consistent to enable accurate age prediction with

\footnotetext{
*Correspondence: tim.jenkins@hsc.utah.edu

${ }^{1}$ Andrology and IVF Laboratories, University of Utah, 675 Arapeen Dr. Suite 201, Salt Lake City, UT 84108, USA

Full list of author information is available at the end of the article
}

Horvath's age calculator despite the significant contrast in epigenetic profiles between various somatic tissues.

Despite the general applicability of this model across diverse tissues, one tissue in particular did not display similar predictive power as was seen with most. In fact, DNA methylation signatures from testicular tissue and sperm specifically did not appear to be predictive of age at all with the previously described calculator [1]. In agreement with this observation is data from our lab which suggests that the nature of age associated alterations to sperm DNA methylation signatures are opposite of what is typically seen in somatic cells [1-4]. Specifically, although aging results in a global decrease in methylation and increased regional methylation in most cell types, we demonstrated that sperm exhibits the opposite trend. In many ways such a finding is not surprising as this is not the first case where the male 
germ line defied conventional age-associated cellular alterations. The most well described example of this is that of age impacts on telomere length. A hallmark of aging in somatic cells is a marked shortening of telomeres, but in sperm telomere lengthening is commonly seen with aging [5]. Clearly, sperm cells are extraordinarily unique and thus it seems likely that a unique approach is required to understand both the nature of the aging process and the potential predictive power of age associated alterations to the sperm epigenome.

In our previous publications we have described the general impact of aging on the sperm methylome. In these studies, we have shown that sperm have a very distinct pattern of age-associated alteration [2, 3]. We identified 148 genomic regions $(\sim 1 \mathrm{~kb}$ in size) that displayed differential methylation with age. Of these, only 8 displayed an increase in methylation, and the remaining 140 regions experienced a marked loss of methylation with age. Intriguingly, these regions of differential methylation are enriched at genes known to be associated with bipolar disorder and schizophrenia, both diseases known to have increased incidence in the offspring of older fathers. Indeed the epigenetic patterns of aging in sperm, while distinct from the epigenetic patterns of aging in somatic tissues, are striking and extremely consistent and thus provide an excellent opportunity for predictive model construction.

The pursuit of generating a model to predict an individual's age using the sperm methylome is not only an interesting question from the perspective of basic cell biology but the patterns of sperm aging, and the unique nature of the sperm make the utilization of this cell type ideal for such a predictive model. Using pure cell populations is ideal for any epigenetic analysis, and while the previously constructed models are effective at predicting age even with tissues that are difficult to purify (which is a testament to quality of model and to the strength of the aging signal), the ideal scenario would be to use a pure cell population. Human sperm offer just such an opportunity. Many protocols are applied to somatic cell removal in sperm epigenetic studies and they have proven quite effective at isolating only germ cells, thanks in large part to the highly unique and compact nature of the sperm head. Further, the magnitude of the aging signal is quite strong in the sperm (thought to be in part due to the highly proliferative nature of the sperm cells themselves) and as a result, the patterns of aging offer an excellent opportunity for powerful prediction. In this study, we set out to capitalize on these advantages to build a model that can predict an individual's age using methylation signatures in the paternal germ line. The experiments outlined herein describe the utility of the germ line age calculation and also provide evidence to suggest that the rate of aging can be affected by environmental exposures or lifestyles (smoking, obesity, etc.).

\section{Results}

\section{Model construction and training}

In the current study we assessed sperm DNA methylation array data (Illumina $450 \mathrm{~K}$ array) from 3 distinct previously performed studies $[2,6,7]$. From these data sets, we were able to utilize a total of 329 samples that were used to generate the predictive model outlined herein. Individuals with many different fertility phenotypes provided the samples used in this study. Specifically, our training data set includes samples from sperm donors [2], known fertile individuals, infertility patients (including those seeking intrauterine insemination or even in vitro fertilization treatment at our facility), and individuals from the general population [6, 7]. Further, our data set includes those that have very different lifestyles and environmental exposures (as an example, both heavy smokers and never smokers are represented in our data set).

We utilized the glmnet package in $\mathrm{R}$ to facilitate training and development of our linear regression age prediction model [8]. Beta-values were used in all experiments. These values represent fraction methylation as the standard output from the Illumina methylation array, which are scored between 0 and 1 with 0 representing complete demethlyation and 1 representing complete methylation. For training of our model, we first tested multiple designs to generate the most robust and easily interpretable model. We first constructed a model trained on all CpGs on the entire array ("entire array" training). We additionally limited the training dataset to only 148 regions that we have previously identified to be strongly associated with the aging process to ensure the broad interpretability to the results of the model [2]. We trained two models within those 148 genomic regions to identify the best possible outcomes. First, we trained on all of the beta-values for each CpG located in our regions of interest ("CpG level" training). Second, we generated a mean of beta-values for each region that included the CpGs within each region respectively yielding mean beta-values for each region ("regional level" training), and the model was trained only on these averages.

In each of the above-described scenarios, we employed a 10 -fold cross validation strategy. This was performed 10 times on unique subgroups of the entire data set (Fig. 1a-f). The results from these ten validations were compared between the CpG level training and the regional level training. To compare the accuracy and predictive power of these models we performed linear regression for each (actual age vs. predicted age) and generated $R^{2}$ values. These $R^{2}$ values were compared via simple two-tailed t-test to determine if any significant differences exist between the various approaches to model construction (entire array, CpG level construction, regional level construction). These tests revealed that when only considering the prediction results on 

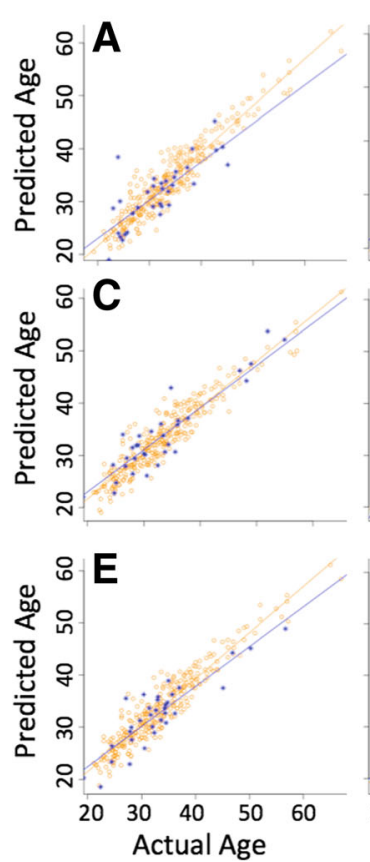
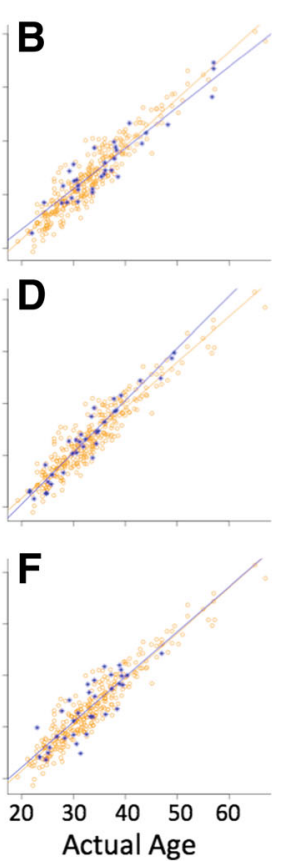

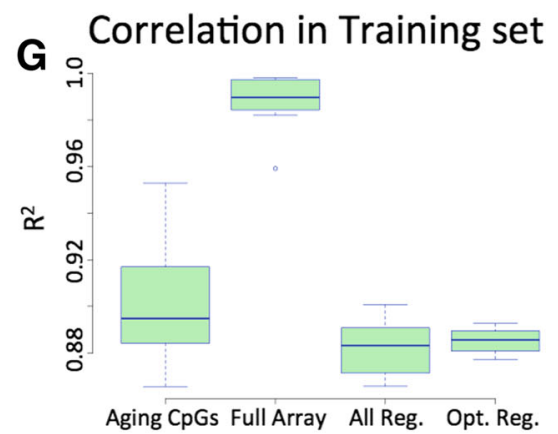

H Correlation in Test set

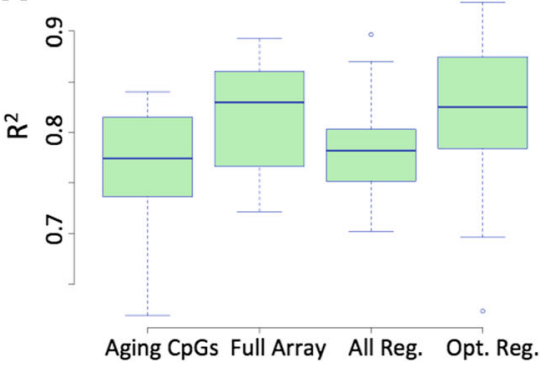

Fig. 1 a-f Scatterplots depicting the relationship between predicted and chronological age in 6 represented models from our cross validation testing. $\mathbf{g}$ Box and whisker plots of the R2 values (predicted vs. actual) for the training data set from each cross validation for all four potential model designs including the CpG level training across the entire array and only those within the age-affected regions, as well as the full regional data set (148 regions) and the optimized regional data set (51 regions). $\mathbf{h}$ Box and whisker plots of the R2 values (predicted vs. actual) for the test data set from each cross validation for all four potential model designs including the CpG level training across the entire array and only those within the age-affected regions, as well as the full regional data set (148 regions) and the optimized regional data set (51 regions)

samples within the training set (those samples upon which the model was trained) there was a significant increase in predicative power when training on the entire array compared to any other approach (Fig. 1g) and that there was a modestly significant decrease in predictive power in the regional model (when compared to the CpG level model; $p=0.0428$ ). However, when applying the predictive model to the test dataset these differences were no longer seen. In fact, there were no significant differences seen between the predictive capacities of the different models in these test sets (Fig. 1h). In an effort to make the model as simple as possible and in light of these findings, we committed to use the regional level model moving forward. Further, the alterations that occur at single CpGs are less likely to be biologically meaningful than those that occur over a region of the genome, thus using the regional level feature set improves the biological interpretability.

We additionally assessed the weighting of the features (regions) used in the models constructed during cross validation. We found a great deal of variation in the features selected across the regions screened, though a subset of the regions were heavily weighted and used in $80 \%$ or more of the models built during cross validation (a total of 51 features/regions met this criterion). In an effort to identify the simplest model we compared cross validation (10-fold strategy) in only these 51 regions ("optimized regions") to all of the regions previously screened. We found that both the training and test groups were not statistically different between the optimized regional list and the full regional list (Fig. 1h). Further, the best performing model (and ultimately the selected model from our work) of any we tested was trained only on the optimized list of 51 regions of the genome (Table 1). In the training data set this model performed quite well with an $\mathrm{r}^{2}=0.93$, and similar predictive power was seen when screening all 329 samples in our data set $\left(r^{2}=0.89\right)$. To further highlight the power of prediction of this model it is helpful to note that our model predicted age with a mean absolute error (MAE) of 2.04 years, and a mean absolute percent error (MAPE) of $6.28 \%$ in our data set, thus the average accuracy in prediction is approximately $93.7 \%$.

\section{Technical validation / replicate performance}

Because variability can be a concern in array experiments, we tested our model in a completely independent cohort of samples that were not used in any of our cross validation / model training experiments. We utilized 10 sperm samples, each with 6 replicates (a total of 60 samples) that were each run on the $450 \mathrm{~K}$ array platform from a previously published study [9]. Further, the samples from this 
Table 1 Genomic regions used for age prediction

\begin{tabular}{|c|c|c|c|}
\hline Name & $\mathrm{CHR}$ & Start & Stop \\
\hline ADAMTS8 & chr11 & $130,299,298$ & $130,299,948$ \\
\hline ARC & chr8 & $143,694,010$ & $143,694,548$ \\
\hline ARGHGEF10 & chr8 & $1,877,888$ & $1,878,324$ \\
\hline BCL11A & chr2 & $60,680,616$ & $60,680,762$ \\
\hline C1ORF122 & chr1 & $38,272,200$ & $38,273,057$ \\
\hline C7ORF50 & chr7 & $1,083,209$ & $1,084,163$ \\
\hline CCDC144NL & chr17 & $20,798,895$ & $20,799,770$ \\
\hline CLIC1 & chr6 & $31,698,492$ & $31,699,299$ \\
\hline DMPK & chr19 & $46,282,571$ & $46,283,081$ \\
\hline FAM86C1 & chr11 & $71,498,202$ & $71,499,118$ \\
\hline FAM86JP & chr3 & $125,634,060$ & $125,634,453$ \\
\hline FOXK1 & chr7 & $4,722,778$ & $4,723,928$ \\
\hline $\mathrm{FSCN}$ & chr7 & $5,635,134$ & $5,635,954$ \\
\hline GAPDH & chr12 & $6,641,602$ & $6,642,355$ \\
\hline GET4 & chr7 & 914,964 & 915,832 \\
\hline GNB2 & chr7 & $100,274,361$ & $100,275,305$ \\
\hline GPANK1 & chr6 & $31,630,819$ & $31,632,542$ \\
\hline GPR45 & chr2 & $105,857,809$ & $105,859,084$ \\
\hline KCNQ1 & chr11 & $2,554,562$ & $2,555,577$ \\
\hline LDLRAD4 & chr18 & $13,611,370$ & $13,611,825$ \\
\hline LMO3 & chr12 & $16,760,040$ & $16,761,003$ \\
\hline LOC100133461 & chr4 & $3,680,721$ & $3,681,760$ \\
\hline MIR22HG & chr17 & $1,617,363$ & $1,618,296$ \\
\hline MTMR8 & $\operatorname{chr} x$ & $63,614,857$ & $63,615,496$ \\
\hline N10 & chr1 & $28,423,399$ & $28,424,202$ \\
\hline N12 & chr5 & $3,593,413$ & $3,594,276$ \\
\hline N22 & chr19 & $4,579,481$ & $4,580,471$ \\
\hline N23 & chr14 & $106,004,434$ & $106,004,608$ \\
\hline N24 & chr6 & $170,449,417$ & $170,450,804$ \\
\hline N27 & chr6 & $30,432,200$ & $30,433,944$ \\
\hline N30 & chr15 & $27,959,473$ & $27,960,032$ \\
\hline N8 & chr11 & $69,260,136$ & $69,261,045$ \\
\hline N9 & chr7 & $35,300,077$ & $35,301,070$ \\
\hline NCOR2 & chr12 & $124,990,897$ & $124,991,140$ \\
\hline NONE & chr10 & $17,347,047$ & $17,347,392$ \\
\hline NSG1 & chr4 & $4,386,726$ & 4,387,698 \\
\hline PAX2 & chr10 & $102,509,693$ & $102,510,569$ \\
\hline PITX1 & chr5 & $134,365,728$ & $134,366,535$ \\
\hline PRSS22 & chr16 & $2,908,157$ & $2,908,935$ \\
\hline PTPRN2.3 & chr7 & $157,523,356$ & $157,524,159$ \\
\hline PTPRN2.4 & chr7 & $158,109,339$ & $158,110,153$ \\
\hline PURA & chr5 & $139,492,535$ & $139,493,491$ \\
\hline PYY2 & chr17 & $26,553,567$ & $26,554,908$ \\
\hline SECTM1 & chr17 & $80,278,592$ & $80,280,331$ \\
\hline
\end{tabular}

Table 1 Genomic regions used for age prediction (Continued)

\begin{tabular}{llll}
\hline Name & CHR & Start & Stop \\
\hline SEMA6B & chr19 & $4,555,999$ & $4,556,983$ \\
SEZ6 & chr17 & $27,330,794$ & $27,332,647$ \\
SLC22A18AS & chr11 & $2,909,690$ & $2,909,716$ \\
SOHLH1 & chr9 & $138,590,204$ & $138,590,996$ \\
THBS3 & chr1 & $155,176,868$ & $155,177,784$ \\
TNXB & chr6 & $32,064,146$ & $32,065,891$ \\
\hline
\end{tabular}

study were exposed to varying extremes in temperature to test the stability of the sperm DNA methylation signatures. Thus these samples do not represent strict technical replicates (because of slight variations in treatment) but do provide an even more robust test of the algorithms predictive power on sperm DNA methylation signatures in multiple samples from the same individual. The model was applied to these samples and performed well in both precision and accuracy. Specifically, not only was the consistency of predictions in this independent cohort quite robust ( $\mathrm{SD}=0.877$ years), but the accuracy of prediction was very similar to what was seen in the training data set with an MAE of 2.37 years (compared to 2.04 years in the training data set) and a MAPE of $7.05 \%$ (compared to $6.28 \%$ in our training data set). We additionally performed linear regression analysis on the predicted age vs. actual age in each of the 10 individuals in the dataset and found a significant association between these two $\left(\mathrm{R}^{2}\right.$ of $0.766 ; p=0.0016$; Fig. 2 ).

\section{The impact of smoking on age prediction}

To test the potential diagnostic/clinical utility of our model we have more closely assessed the data in our original cross validation dataset. Specifically we have analyzed our smoking dataset [7], which includes sperm methylation data from 78 smokers and 78 individuals who responded as "never smokers." Similar aged men are represented in each group. We additionally isolated a portion of the smoking group who were had smoked cigarettes for $>10$ years. We found an approximately $1.5 \%$ increased in predicted age compared to chronological age in all smokers and $2.5 \%$ increase in long term smokers. However this difference failed to reach statistical significance. Interestingly, this same pattern was observed (though significantly higher in magnitude) when screening only individuals who were less than 35 years old at the time of collection (Fig. 3). In these samples we saw a $3 \%$ increase in predicted age compared to chronological age in the smoker group and a nearly $6 \%$ increase in predicted age in the long-term smokers $(p=0.0196)$.

\section{Discussion}

We have developed a sperm age calculator that has the capacity to identify an individual's chronological age 


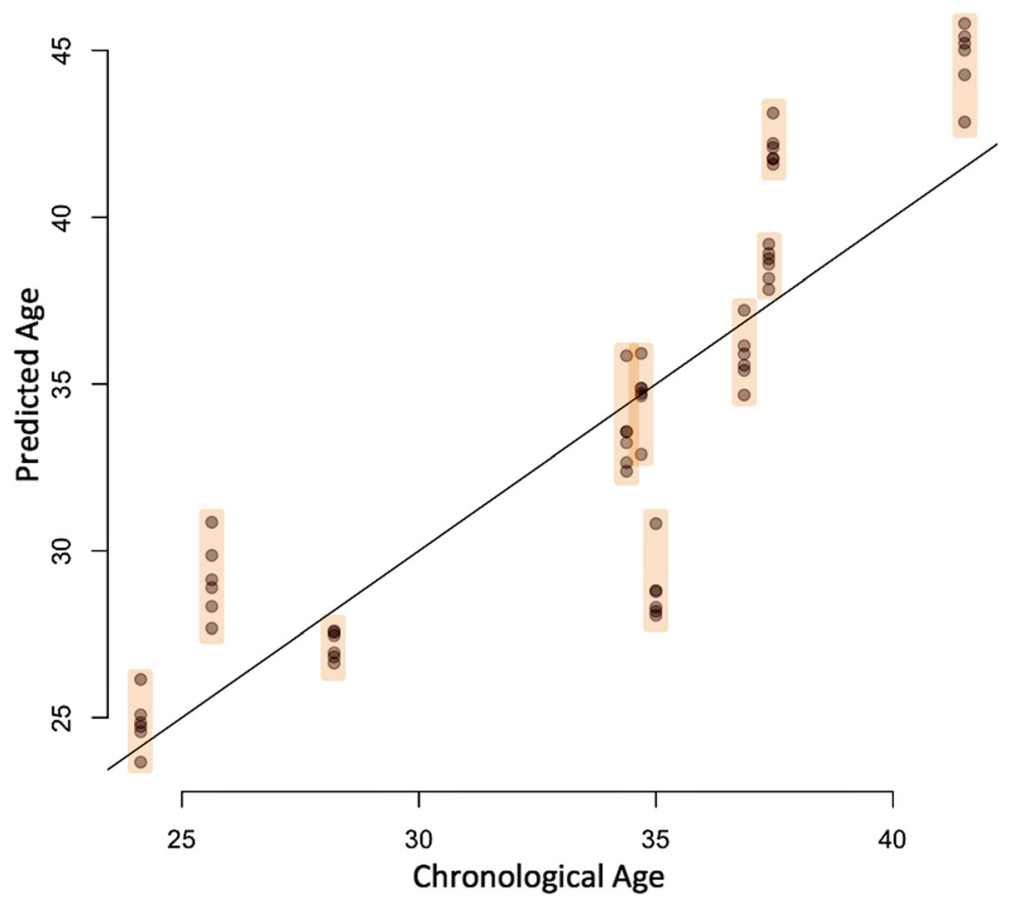

Fig. 2 Scatterplot depicting the age prediction in a completely independent cohort of 10 samples each of which has 6 technical replicates. The points within each discrete orange box represent predictions for all six replicates from each individual. The line is representative of a "perfect" prediction

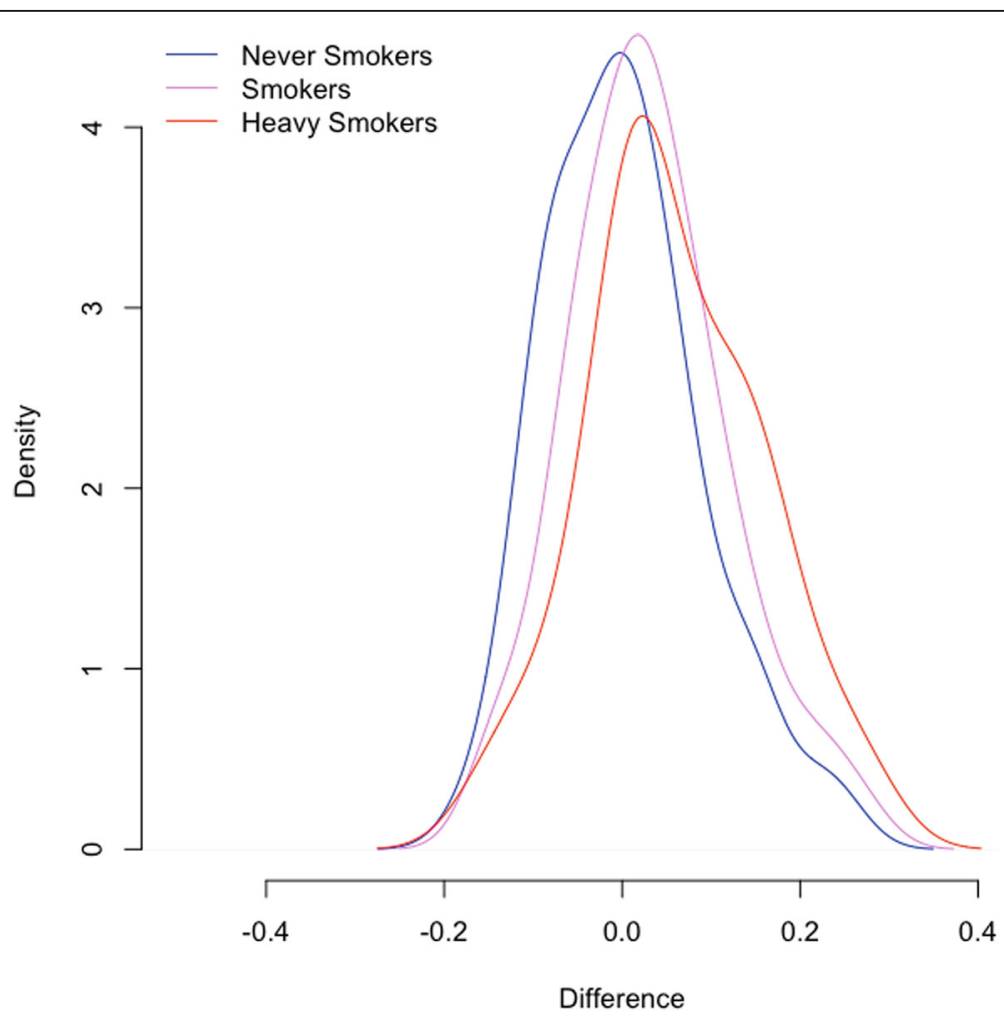

Fig. 3 Density plot shows the accuracy of age prediction in never smokers, smokers, and heavy smokers among individuals below 35 years of age. Similar patterns exist in the entire cohort but are the most profound in this age group 
based only on their sperm DNA methylation signatures. Previous studies have defined aging patterns in somatic cells and one in particular [1] very successfully generated an aging calculator using methylation signatures of somatic cells. However, these findings do not hold true in sperm and further, the DNA methylation age calculator that described in 2013 fails to work effectively with paternal germ line epigenetic signatures. Herein, we have described the development of a linear model that has the ability to accurately predict ages with these signatures. Specifically our model is based on average methylation signatures at 51 genomic loci known to be altered as men age [2].

In the process of model construction, we evaluated multiple potential methods by which we could train our model. One important consideration was the nature of the population with which the model was trained. While there is a balance in selecting a population (broad applicability vs. targeted population) we decided to utilize a population with diverse fertility phenotypes and exposures to ensure that it could perform well with many different phenotypes. As such we included smokers and non-smokers, individuals of known fertility, those currently being treated for infertility, and men from our general population.

We also attempted to obtain a simple model with the fewest number of features needed to achieve optimal predicative power. Our initial approach was to focus only on the regions previously identified to be altered by age (based on previous data) and refined the model by only assessing these regions. The rational behind this approach stems from the understanding the changes to methylation signatures occurring in small regions of the genome (at promoters and CpG islands) have the potential to affect transcription and thus phenotype [10]. Thus, using fraction methylation across an entire region that has already been demonstrated to be associated with aging in sperm offers an approach that is likely the most directly tied to biological function. In fact, our own previous studies assessing these specific aging marks suggest that they may have biologic significance in the offspring of older males [2]. We compared models trained with this restricted feature set to models built using all available data (the entire array) as features. While the models built with the entire array did have increased predictive power when testing them in the same samples that were used for model training, there was no difference seen when predicting ages in a test group (to which the model was blinded during construction) between models built using the restricted and regionalized feature set and the whole array feature set. Importantly, the most efficient single model (considering only data from tests using samples that had not been used in training) was constructed using the restricted and regionalized feature set. The fact that models built using the entire array had improved performance in the training set without any improvement in the test set supported our approach to use a simplified and more restricted set of features for training. This is because when using the entire array, model construction was able to identify some features that appeared to be predictive of age but these were, in reality, only effective predictors in the samples used in training and thus added no benefit when training in samples that had not been used in model construction. We found that even in our model using regional level features there was some amount of simplification that could be performed. Indeed, we were able to scale our list of features from 148 regions down to 51 regions with the same predictive power. This effort resulted in a quite robust model with strong predictive power (an average of $~ 93 \%$ accuracy in predicting each individual's age and an $\mathrm{r}^{2}$ of $\sim 0.89$ ).

Our data indicate that the model constructed herein is also technically robust. We were able to assess previous data from our lab in which 10 individuals had six technical replicates on $450 \mathrm{k}$ methylation arrays [9]. This replicate data enabled to assess the power of the model in two distinct ways. First, we were able to assess the predictive power of the model on a completely independent cohort (each of these samples were assessed at a different time and in different batches of arrays than those upon which the model was trained). Second, we were able to show that the model is able to generate consistent predictions for individuals between technical replicates. Of additional interest is the fact that the samples used in these technical replicates originated from a study that tested the impact of extreme and prolonged temperature exposures on sperm DNA methylation patterns. Thus a portion of the replicates screened were exposed to various magnitudes of less than ideal conditions. However, it is important to note that we did observe a drop in $r^{2}$ in our independent cohort. This is not an entirely unexpected finding due to the variation that can occur between different batches of arrays. In brief, we found that the array batch effects are sufficiently strong to slightly decrease the predictive power of the age calculator for batches performed outside of our original training set. In contrast, the model is sufficiently strong to overcome such variation with strong predictive power, though that power is slightly reduced compared to what is seen in our training/test data set. The ability to maintain predictive power, even when assessing other batches of data is important in a model that will have broad applicability. In the future as more data become available, the model can be updated with increased sample size and with additional batches of experiments, which will lead to even more robust predictive power.

It is important to note that due to the difficulty in obtaining samples for very young individuals and older individuals (those outside of the typical age of paternity) our model is constructed mainly using samples in men 
between 20 and 45 years of age. As a result, we expect that moving forward when samples from older men become available that the model should be updated to include more samples from men of different ages to improve the predictive power at other ages.

Our data also suggest that there may be some utility for such a model in a clinical setting. Specifically, we were able to identify an age-affect of smoking in our cohort of patients. We found that individuals who smoke appeared to have acceleration in the pattern of aging and thus the individual's germ line age was in some cases significantly higher than their chronological age. However, this finding specifically should be taken in the proper context, where we only identified significant age acceleration in a portion of the data set and that (though all age ranges followed the same trend) the magnitude of the effect varied greatly. Still, this represents one example of many different analyses that could be performed in a clinical setting should further exploration identify consistent and impactful age acceleration. With future studies we may find that different levels/types of infertility, obesity, or other environmental exposures may cause acceleration in the aging pattern seen in sperm. One of the biggest questions that remains if such associations exist is the potential impact of this age acceleration. Such a pattern could potentially result in increased risk to offspring health, as epidemiological data clearly shows increased incidence of neuropsychiatric disease in the offspring of older fathers [1116]. This increase in risk may not mean that the altered methylation pattern itself causes these offspring abnormalities, but instead the methylation signatures of age are simply a good indicator of the overall state or age of the sperm. Likely of more immediate interest to clinicians is the fact that advanced paternal age is associated with a loss of fecundity and fertility. Specifically, it has been shown that men older than 45 years take $\sim 5$ times longer to achieve a pregnancy as men less than 25 years (when controlling for female age) [17]. A similar decrease in fecundity was identified in a large population study in 2000 which showed that (after adjusting for maternal age) men $>35$ years of age had a 50\% lower chance of achieving a pregnancy within 12 months of attempting conception than younger men [18]. Other studies have also shown decreased fertilizing potential in both IUI and IVF [19, 20]. While the magnitude of this effect remains controversial $[21,22]$, it is clear that advanced paternal age does play an important role in a couple's fertility status and can clearly result in, at a minimum, a significantly increased time to pregnancy. For many couples, such potential barriers to achieving a pregnancy are essential to understand and discuss with their care providers. While none of these associations have been proven in this specific work, the potential clinical utility of the calculator is clear and warrants further investigation both in predicting an individual's health/fertility as well as in the prediction of abnormalities in the offspring.

It is important to note that while the findings of alterations associated with age in the sperm epigenome are intriguing, the direct impact of these alterations is still in question. In fact, the actual impact of any sperm epigenetic alteration on the embryo or the offspring is difficult to predict due to massive reprograming events that take place in the early embryo and in the primordial germ cells. However, data do suggest that methylation marks in many sub-telomeric regions escape reprograming events and can be potentially be passed on to the offspring [23-27]. Intriguingly, our original sperm aging study showed that the majority of age-affected regions were located in these sub-telomeic regions as well [2]. Such a transmission of age-affects would be remarkable, but may offer a real potential explanation for at least a portion of the downstream impact of paternal age on offspring disease incidence and phenotype.

The data described herein are quite promising, though some limitations are clear. Foremost among them is our knowledge of downstream impacts as described above. This will require a great degree of effort to determine the nature of these effects and if risks to fertility or the offspring can be modified in any way by various treatments. Further, while the current model is very effective at predicting an individuals age and is quite robust technically, the alterations we are observing to predict age are subtle and thus small inefficiencies can result in an inability to detect meaningful changes. Despite this, because of the approach we have taken in designing a model based only on limited numbers of regions there is a potential to modify this model for use with different platforms that may offer increased resolution and consistency, for example targeted sequencing [28]. With such an approach, we may be able to improve an already robust predictive model by multiplex sequencing with extreme depth at only the 51 sites of interest. This could provide an even more economical and reliable predictive model. Taken together, the data that we have shown here are intriguing and warrant a great deal of further investigation and also have the potential to be improved with future iterations.

\section{Conclusions}

Similar to what is seen in somatic cells, our data clearly indicate that sperm methylation signatures are altered over time and that these modifications can be used to predict an individual's age. This enabled the development of a germ line age calculator that is presented in this work. This calculator offers tremendous potential in the basic sciences to address fundamental questions about aging, fertility, and potential impacts on embryogenesis and even offspring health. In addition, while much work is yet required, it appears that such calculations may have 
potential benefit in clinical fertility care as well as in forensics. While the future potential is evident, the promise of the technology and its biological underpinnings will not be fully realized without significant future efforts in both animal models and in direct human studies.

\section{Methods}

\section{Samples, study design, data availability}

In the current study we assessed sperm DNA methylation array data from 3 distinct previously performed studies $[2,6,7]$. All of the studies were performed in our laboratory. We included only the samples for which ages were available. From these data sets, we were able to acquire a total of 329 samples that were used to generate the predictive model outlined herein. Each sample was run on the Illumina $450 \mathrm{~K}$ methylation array. In each case, we used SWAN normalization to generate beta-values (values between 0 and 1 that represent the fraction of a given CpG that is methylated) that were used in our study. During early processing of the sperm samples, great care was taken to ensure that no somatic cell contamination was present that could potentially influence the results of our studies. To confirm the absence of somatic cell contamination we assessed the methylation signatures at a number of sites throughout the genome, each of which are highly differentially methylated between sperm and somatic tissues. In Fig. 4, we show the differential methylation at one representative genomic locus, DLK1, to illustrate the absence of contaminating signals in the samples used in our study.

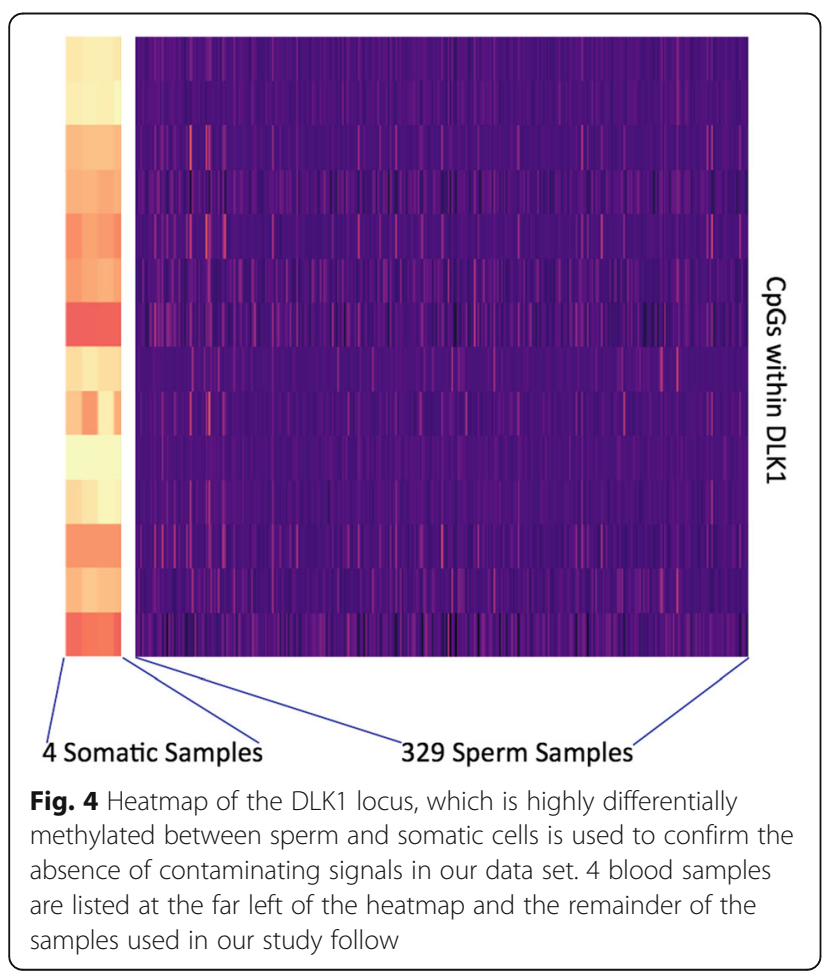

While variability exists between the methylation in these samples there exists very little, if any somatic DNA methylation signals.

\section{Samples used}

Individuals with many different fertility phenotypes provided the samples used in this study. Our training data set includes samples from sperm donors, known fertile individuals, infertility patients (including those seeking intrauterine insemination or even in vitro fertilization treatment at our facility), and individuals from the general population. Further, our data set includes those that have very different lifestyles and environmental exposures (heavy smokers and never smokers, Obese individuals and those with normal BMIs, etc.).

The average ages in each study were statistically similar (with averages of approximately 33 years of age) aside from the smallest study used [2], which previously assessed aging patterns (average age of approximately 44 years of age). Known fertile sperm donors collected $\sim 27 \%$ of all samples used in the study. Individuals from the general population in the Salt Lake City area collected $31 \%$ of the samples and infertility patients collected another $42 \%$ of the samples used in the study. Of all the individuals included in our study approximately $26 \%$ are smokers. In terms of BMI, $46 \%$ of the men in our study were considered normal, $35 \%$ were considered overweight, and $9 \%$ were classified as obese.

\section{Model training}

We utilized the glmnet package in $\mathrm{R}$ to facilitate training and development of our linear regression age prediction model [8]. For training of our model, we first tested multiple designs to generate the most robust and easily interpretable model. We first constructed a model trained on all CpGs on the entire array ("entire array" training). We additionally limited the training dataset to only 148 regions that we have previously identified to be strongly associated with the aging process to ensure the broad interpretability to the results of the model [2]. We trained two models within those 148 genomic regions to identify the best possible outcomes. First, we trained on all of the beta-values for each $\mathrm{CpG}$ located in our regions of interest ("CpG level" training). Second, we generated a mean of beta-values for each region that included the CpGs within each region respectively yielding mean beta-values for each region ("regional level" training), and the model was trained only on these averages.

In both of the above-described scenarios, we employed a 10-fold cross validation strategy to repeatedly test trainings on $90 \%$ of our samples and hold out $10 \%$ for a test set. This was performed 10 times on unique subgroups of the entire data set. The results from these ten validations were compared between the entire array 
training, the CpG level training, and the regional level training. To compare the accuracy and predictive power of these models we performed linear regression for each (actual age vs. predicted age) and generated $\mathrm{r}^{2}$ values. These $r^{2}$ values were compared via simple two-tailed t-test to determine if any significant difference exists between the two approaches to model construction $(\mathrm{CpG}$ level construction vs. regional level construction).

\section{Technical validation / replicate performance}

We tested our model in a completely independent cohort of samples [9]. We used 10 sperm samples each with six technical replicates that were each run on the $450 \mathrm{~K}$ array (not those used in our cross validation / model training) to determine the precision and consistency of prediction. These samples were all taken from men who were attending the Andrology lab for a fertility workup. In each case the men who provided the sample had normal semen analysis measures. Linear regression analysis of predicted vs. actual age was performed using $\mathrm{R}$.

\section{The impact of smoking on age prediction}

We tested 78 never smokers and 78 smokers using our age prediction model. Similar aged men are represented in each group. We additionally isolated a portion of the smoking group who have smoked cigarettes for $>10$ years. In this analysis we compared accuracy of the age prediction of each group to determine if there is a significant increase in the age prediction compared to chronological age in individuals who smoke. We identified the percent difference between chronological age and predicted age and compared this value between smokers and non-smokers via two-tailed t-test to identify the presence of age acceleration.

\section{Acknowledgments}

We recognize the efforts of Chris Conley from the Huntsman Cancer Institute for his technical assistance.

\section{Funding}

A portion of the data used in this manuscript originated from work performed in our lab, which was funded by the NIH (RO1HD082062).

\section{Availability of data and materials}

The model generated in this manuscript as well as all instructions on use are publically available on a publically available repository:

Jenkins, TG, Sperm Aging Calculator, (2017), GitHub repository, https:// github.com/timgjenkins/Jenkins-et-al-2017

\section{Authors' contributions}

TJ performed all computational work and designed the study. KA assisted in bioinformatics and study design. AS provided guidance regarding the implementation of machine learning techniques and study design. DC and $\mathrm{BC}$ provided oversight and suggestions for implementation. All authors read and approved the final manuscript.

\section{Ethics approval and consent to participate}

Our study was assessed and approved by the institutional review board at the University of Utah and all patients provided written informed consent to participate.

\section{Consent for publication \\ Not Applicable.}

\section{Competing interests}

$T J, K A, B C$, and DC are listed as inventors on IP held by the University of Utah associated with aging and sperm epigenetic patterns. DC and AS hold equity in a company, Episona, which is developing tools for male infertility diagnosis using epigenetic signatures.

\section{Publisher's Note}

Springer Nature remains neutral with regard to jurisdictional claims in published maps and institutional affiliations.

\section{Author details}

${ }^{1}$ Andrology and IVF Laboratories, University of Utah, 675 Arapeen Dr. Suite 201, Salt Lake City, UT 84108, USA. ${ }^{2}$ Huntsman Cancer Institute, Salt Lake City, USA. ${ }^{3}$ University of Southern California, Los Angeles, USA. ${ }^{4}$ Department of Obstetrics and Gynecology, University of Utah, Salt Lake City, USA.

${ }^{5}$ Department of Genetics, University of Utah, Salt Lake City, USA.

Received: 3 April 2018 Accepted: 9 October 2018

Published online: 22 October 2018

References

1. Horvath S. DNA methylation age of human tissues and cell types. Genome Biol. 2013:14(10):R115.

2. Jenkins TG, Aston Kl, Pflueger C, Cairns BR, Carrell DT. Age-associated sperm DNA methylation alterations: possible implications in offspring disease susceptibility. PLoS Genet. 2014;10(7):e1004458.

3. Jenkins TG, Aston Kl, Cairns BR, Carrell DT. Paternal aging and associated intraindividual alterations of global sperm 5-methylcytosine and 5hydroxymethylcytosine levels. Fertil Steril. 2013:100(4):945-51.

4. Richardson B. Impact of aging on DNA methylation. Ageing Res Rev. 2003; 2(3):245-61.

5. Allsopp RC, Vaziri H, Patterson C, Goldstein S, Younglai EV, Futcher AB, Greider CW, Harley CB. Telomere length predicts replicative capacity of human fibroblasts. Proc Natl Acad Sci U S A. 1992:89(21):10114-8.

6. Aston KI, Uren PJ, Jenkins TG, Horsager A, Cairns BR, Smith AD, Carrell DT: Aberrant sperm DNA methylation predicts male fertility status and embryo quality. Fertil Steril 2015, 104(6):1388-1397 e1381-1385

7. Jenkins TG, James ER, Alonso DF, Hoidal JR, Murphy PJ, Hotaling JM, Cairns BR, Carrell DT, Aston Kl. Cigarette smoking significantly alters sperm DNA methylation patterns. Andrology. 2017:5(6);1089-99.

8. Friedman J, Hastie T, Tibshirani R. Regularization paths for generalized linear models via coordinate descent. J Stat Softw. 2010;33(1):1-22.

9. Jenkins TGSM, James E, Aston KI, Carrell DT. Thermo stability of DNA methylation marks in human sperm. J Genet Genome Res. 2017:4(1)

10. Deaton AM, Bird A. CpG islands and the regulation of transcription. Genes Dev. 2011:25(10):1010-22.

11. Frans EM, Sandin S, Reichenberg A, Langstrom N, Lichtenstein P, McGrath Jر J Hultman CM. Autism risk across generations: a population-based study of advancing grandpaternal and paternal age. JAMA Psychiatry. 2013;70(5): 516-21.

12. Idring S, Magnusson C, Lundberg M, Ek M, Rai D, Svensson AC, Dalman C, Karlsson $\mathrm{H}$, Lee BK. Parental age and the risk of autism spectrum disorders: findings from a Swedish population-based cohort. Int J Epidemiol. 2014; 43(1):107-15.

13. Miller B, Messias E, Miettunen J, Alaraisanen A, Jarvelin MR, Koponen $H$, Rasanen $\mathrm{P}$, Isohanni M, Kirkpatrick B. Meta-analysis of paternal age and schizophrenia risk in male versus female offspring. Schizophr Bull. 2011; 37(5):1039-47.

14. Naserbakht M, Ahmadkhaniha HR, Mokri B, Smith CL. Advanced paternal age is a risk factor for schizophrenia in Iranians. Ann General Psychiatry. 2011;10:15.

15. Dalman C. Advanced paternal age increases risk of bipolar disorder in offspring. Evid Based Ment Health. 2009;12(2):59. 
16. Kuratomi G, Iwamoto K, Bundo M, Kusumi I, Kato N, Iwata N, Ozaki N, Kato T. Aberrant DNA methylation associated with bipolar disorder identified from discordant monozygotic twins. Mol Psychiatry. 2008;13(4):429-41.

17. Hassan MA, Killick SR. Effect of male age on fertility: evidence for the decline in male fertility with increasing age. Fertil Steril. 2003;79(Suppl 3):1520-7.

18. Ford WC, North K, Taylor H, Farrow A, Hull MG, Golding J. Increasing paternal age is associated with delayed conception in a large population of fertile couples: evidence for declining fecundity in older men. The ALSPAC study team (Avon longitudinal study of pregnancy and childhood). Hum Reprod. 2000;15(8):1703-8.

19. Mathieu C, Ecochard R, Bied V, Lornage J, Czyba JC. Cumulative conception rate following intrauterine artificial insemination with husband's spermatozoa: influence of husband's age. Hum Reprod. 1995;10(5):1090-7.

20. Dain $L$, Auslander R, Dirnfeld M. The effect of paternal age on assisted reproduction outcome. Fertil Steril. 2011;95(1):1-8.

21. Niederberger C. Re: male biological clock: a critical analysis of advanced paternal age. J Urol. 2016;195(3):717.

22. Ramasamy R, Chiba K, Butler P, Lamb DJ. Male biological clock: a critical analysis of advanced paternal age. Fertil Steril. 2015;103(6):1402-6.

23. Guibert S, Forne T, Weber M. Global profiling of DNA methylation erasure in mouse primordial germ cells. Genome Res. 2012;22(4):633-41.

24. Hajkova P, Erhardt S, Lane N, Haaf T, El-Maarri O, Reik W, Walter J, Surani MA. Epigenetic reprogramming in mouse primordial germ cells. Mech Dev. 2002;117(1-2):15-23.

25. Franklin TB, Russig H, Weiss IC, Graff J, Linder N, Michalon A, Vizi S, Mansuy IM. Epigenetic transmission of the impact of early stress across generations. Biol Psychiatry. 2010;68(5):408-15.

26. Popp C, Dean W, Feng S, Cokus SJ, Andrews S, Pellegrini M, Jacobsen SE, Reik W. Genome-wide erasure of DNA methylation in mouse primordial germ cells is affected by AID deficiency. Nature. 2010;463(7284):1101-5.

27. Schmitz RJ, Schultz MD, Lewsey MG, O'Malley RC, Urich MA, Libiger O, Schork NJ, Ecker JR. Transgenerational epigenetic instability is a source of novel methylation variants. Science. 2011;334(6054):369-73.

28. Varley KE, Mitra RD. Bisulfite patch PCR enables multiplexed sequencing of promoter methylation across cancer samples. Genome Res. 2010;20(9):1279-87.

Ready to submit your research? Choose BMC and benefit from:

- fast, convenient online submission

- thorough peer review by experienced researchers in your field

- rapid publication on acceptance

- support for research data, including large and complex data types

- gold Open Access which fosters wider collaboration and increased citations

- maximum visibility for your research: over $100 \mathrm{M}$ website views per year

At $\mathrm{BMC}$, research is always in progress.

Learn more biomedcentral.com/submissions 